\title{
Transmission characteristics of a Fabry-Perot etalon-microtoroid resonator coupled system
}

\author{
Wei Liang, Lan Yang, Joyce K. S. Poon, Yanyi Huang, Kerry J. Vahala, and Amnon Yariv \\ Department of Applied Physics, California Institute of Technology, Pasadena, California 91125
}

Received September 26, 2005; accepted October 30, 2005; posted November 16, 2005 (Doc. ID 65004)

The transmission spectra of a Fabry-Perot etalon coupled to a microtoroid resonator are studied theoretically and experimentally. The resonance line shapes depend strongly on the resonance wavelength detuning and coupling strength between the two resonators. A wide variety of line shapes, ranging from a single to triple peaks, symmetric to asymmetric Fano-like peaks, and notches were predicted and observed experimentally. The capability to modify the spectral line shapes by tuning the coupling between or losses of two resonators may find applications in optical filtering, switching, sensing, and dispersion engineering. () 2006 Optical Society of America

OCIS codes: $230.5750,120.7000,060.2310$.

Coupled oscillators are fundamental to many fields of physics and engineering. Whereas there are many physical manifestations of coupled oscillators, the modes and resonance spectral features of the composite system strongly depend on the details of the constituent oscillators and the coupling mechanism. Thus, coupled optical microresonators may exhibit a variety of spectral responses, depending on the specific resonator configurations of coupling mechanisms. The novel spectral properties of coupled microresonators are potentially applicable to chip-scale optical filters, switches, modulators, and sensors. ${ }^{1-5}$

In this Letter we present theoretically and experimentally the transmission through a Fabry-Perot (FP) etalon $\left(Q \sim 10^{5}\right)$ that is coupled to a highquality-factor $\left(Q>10^{6}\right)$ microtoroid resonator. ${ }^{6}$ By changing the detuning of the resonance wavelengths and varying the coupling strength between the two resonators, the transmission spectrum can be significantly modified. A wide variety of resonance spectral features ranging from single to triple resonance peaks, and from symmetric to asymmetric Fano-like shapes, were predicted theoretically and observed experimentally.
Figure 1 is a schematic description of the coupled microtoroid-FP resonator system, which consists of two fiber Bragg gratings (FBGs), a tapered waveguide, and the microtoroid resonator. This composite system can be modeled as a four-port element whose scattering properties can be described by a $2 \times 2$ ma$\operatorname{trix}_{7} M$, relating the output fields to the input fields, i.e.,

$$
\left(\begin{array}{c}
a_{\text {out }} \\
b_{\text {out }}
\end{array}\right)=M\left(\begin{array}{c}
a_{\text {in }} \\
b_{\text {in }}
\end{array}\right)
$$

where $a_{\text {in }}, b_{\text {in }}, a_{\text {out }}$, and $b_{\text {out }}$ are labeled in Fig. 1 . Each of the constituent elements (i.e., the FBGs, waveguides, and microtoroid) can be modeled in the same way, and $M$ is the product of the scattering matrices representing these individual elements, i.e., $M=M_{\mathrm{FBG}} M_{L} M_{\mathrm{WGM}} M_{L} M_{\mathrm{FBG}}$, where $M_{\mathrm{FBG}}, M_{L}$, and $M_{\text {WGM }}$ are the scattering matrices of the FBG, the waveguides, and the microtoriod, respectively.

Adopting the convention that $a_{0} \exp (i \omega t-i \beta x)$ represents a wave propagating in the $+x$ direction yields the scattering matrices 8,9

$$
\begin{gathered}
M_{\mathrm{FBG}}=\left[\begin{array}{cc}
\cosh \left(s L_{\mathrm{FBG}}\right)-i \delta / s \sinh \left(s L_{\mathrm{FBG}}\right) & -i \zeta / s \sinh \left(s L_{\mathrm{FBG}}\right) \\
i \zeta / s \sinh \left(s L_{\mathrm{FBG}}\right) & \cosh \left(s L_{\mathrm{FBG}}\right)+i \delta / s \sinh \left(s L_{\mathrm{FBG}}\right)
\end{array}\right], \\
M_{L}=\left[\begin{array}{cc}
\exp (-i \beta L) & 0 \\
0 & \exp (i \beta L)
\end{array}\right] \\
M_{\mathrm{WGM}}=\left[\begin{array}{cc}
t_{R} & 0 \\
0 & 1 / t_{R}
\end{array}\right], \quad t_{R}=\frac{\sqrt{1-\kappa^{2}} \exp \left(i \beta^{\prime} 2 \pi R\right)-\alpha}{\exp \left(i \beta^{\prime} 2 \pi R\right)-\sqrt{1-\kappa^{2}} \alpha}
\end{gathered}
$$

In Eq. (2a), $\beta=2 \pi n_{\text {eff }} / \lambda, \delta=\beta-\pi / \Lambda, \zeta=\Delta n \pi / \lambda$, and $s$ $=\left(\zeta^{2}-\delta^{2}\right)^{1 / 2}$, where $\Lambda$ is the grating period and $\Delta n$ is the index contrast, $\lambda$ is the wavelength of light in vacuum, $n_{\text {eff }}$ is the effective modal refractive index, and $L_{\mathrm{FBG}}$ is the length of each FBG. In Eq. (2b), $L$ is the separation between the microtoroid and the FBG. In Eq. (2c), we have assumed that the clockwise (CW) and counterclockwise (CCW) propagating waves in the microtoroid are decoupled in the absence of the FP cavity, leading to the zero off-diagonal terms. $\kappa$ is 


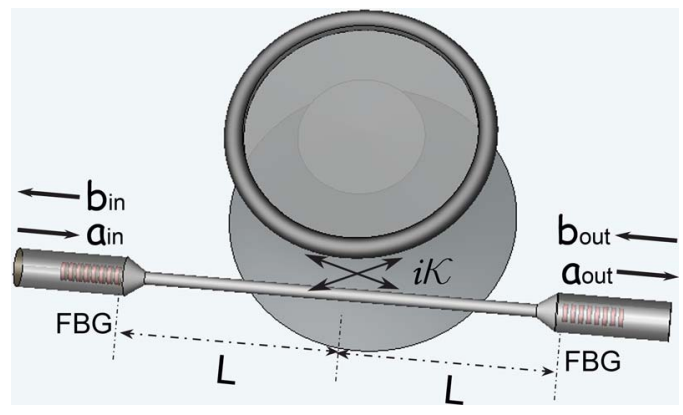

Fig. 1. (Color online) Schematic of a FP etalon coupled to a microtoroid resonator.

a dimensionless coupling coefficient between the microtoroid resonator and the waveguide, as shown in Fig. $1, \beta^{\prime}$ is the propagation constant of the whispering-gallery mode (WGM) in the microtoroid, $\alpha$ and $R$ are the round-trip attenuation factor and the radius of the microtoroid, respectively. For simplicity, we assume that $\beta^{\prime}=\beta$ in the following calculations.

We derive the field amplitude transmission $\left(a_{\text {out }} / a_{\text {in }}\right)$ and reflection $\left(b_{\text {in }} / a_{\text {in }}\right)$ coefficients of the system from $M$ by assuming that no wave is entering the output facet $\left(b_{\text {out }}=0\right)$, such that

$$
\begin{gathered}
b_{\text {in }} / a_{\text {in }}=-M(2,1) / M(2,2), \\
a_{\text {out }} / a_{\text {in }}=[M(1,1) M(2,2)-M(1,2) M(2,1)] / M(2,2) .
\end{gathered}
$$

Using Eqs. (1)-(3), we calculate numerically the transmission and reflection spectra through the FP etalon. In the simulation we choose parameters $n_{\mathrm{eff}}=1.45, \quad \Lambda=0.5238 \mu \mathrm{m}, \quad \Delta_{n}=0.8 \times 10^{-4}, \quad L_{\mathrm{FBG}}$ $=4.0 \mathrm{~mm}, L=3.0 \mathrm{~cm}, R \approx 50 \mu \mathrm{m}, \alpha=0.9998$, and $\lambda$ $\sim 1.520 \mu \mathrm{m}$, similar to our experimental parameters. We categorize our study into two cases: on-resonance or zero detuning between the resonance wavelengths of the two resonators, and off-resonance or detuned resonance wavelengths. Each case can be further divided into undercoupled $\left[\kappa \ll\left(1-\alpha^{2}\right)^{1 / 2}\right]$, intermediately coupled $\left[\kappa \sim\left(1-\alpha^{2}\right)^{1 / 2}\right]$, and overcoupled $[\kappa \gg(1$ $\left.\left.-\alpha^{2}\right)^{1 / 2}\right]$ regimes according to the standard definitions. ${ }^{10}$

Figure 2 shows the variation of the spectral lineshapes on and off resonance for several coupling strengths. In the figures the intensity transmittivities of a single microtoroid resonator, a single FP resonator, and the coupled resonators, as well as the total loss (loss $=1$-transmittivity-reflectivity) are plotted as a function of detuning from the microtoroid resonance, $\lambda_{\mathrm{WGM}}$.

In the undercoupled regime [Figs. 2(a) and 2(e)], the absorptive microtoroid cavity simply provides a narrow intrinsic loss band centered at $\lambda_{\mathrm{WGM}}$. The transmission spectra of the coupled resonators thus exhibit a narrow absorption notch both on and off resonance. In the intermediately coupled regime [Figs. 2(b) and 2(f)], the resonance notches are wider than in the undercoupled regime, indicating that the $Q$ factors are reduced because of the increased coupling. In Fig. 2(b) we have modeled the critical cou- pling case. ${ }^{2}$ The detuned line shape resembles that of a Fano resonance. ${ }^{4,11}$ As the interresonator coupling coefficient is increased to the overcoupled regime, the spectrum acquires a triple-peak shape in the onresonance case [Fig. 2(c)] and a double-peak shape in the off-resonance case [Fig. $2(\mathrm{~g})]$.

An interesting correspondence is possible if we increase the quality factor of the FP resonator when the two resonators are overcoupled. The distorted peaks gradually evolve to separate Lorentzian-type peaks. Specific examples $\left(\Delta n=1.3 \times 10^{-4}\right)$ in the onresonance and the off-resonance cases are plotted in Figs. 2(d) and 2(h). In the on-resonance case the Lorentzian-type peaks can be shown to correspond to the eigenmodes of the coupled system. Specifically, the central peak corresponds to the antisymmetric linear superposition of the microtoroid $(\mathrm{CW}-\mathrm{CCW})$ states and the side peaks correspond to symmetric and antisymmetric linear superposition of the FP resonator mode and the symmetrically paired $(\mathrm{CW}$ $+\mathrm{CCW}$ ) microtoroid mode.

To test the theoretical analysis, we measure the transmission spectra of a coupled microtoroid-FP system for various detuning and interresonator coupling strengths. A silica microtoroid resonator with a diameter of $\sim 50 \mu \mathrm{m}$ and a quality factor of $\sim 2 \times 10^{6}$ is fabricated by use of a combination of lithography, dry etching, and a selective reflow process. The FP resonator is formed by two FBGs written on a hydrogen-loaded Corning SMF-28 fiber by use of a $\mathrm{KrF}$ excimer laser (248 $\mathrm{nm}$ ) and an amplitude phase mask $(\Lambda=524.58 \mathrm{~nm})$. The central part of the FP

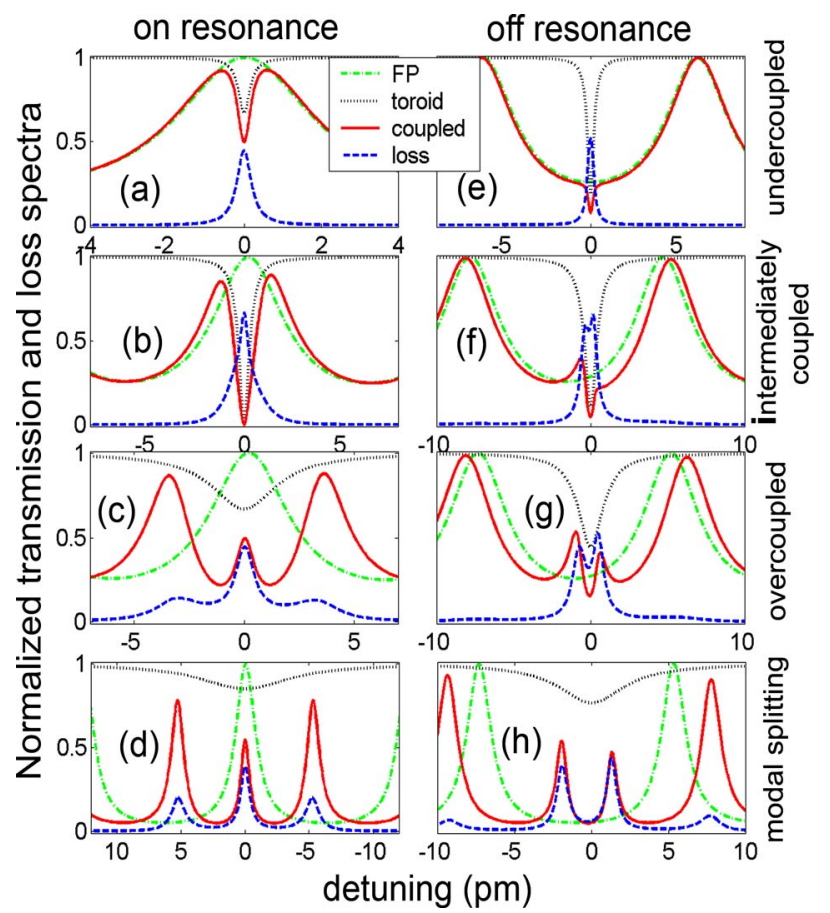

Fig. 2. (Color online) Calculated transmission spectra of a single FP resonator, a single microtoroid resonator, and the coupled-resonator system. Some key parameters that we use in the calculations are $\alpha=0.9998$ and $\kappa=0.0063,0.02$, $0.0632,0.0999,0.0126,0.0283,0.0447,0.0774$, respectively in (a), (b), (c), (d), (e), (f), (g), (h). $\Delta n=0.8 \times 10^{-4}$ in (a)-(c) and (e)-(g); and $\Delta n=1.3 \times 10^{-4}$ in (d) and (h). 


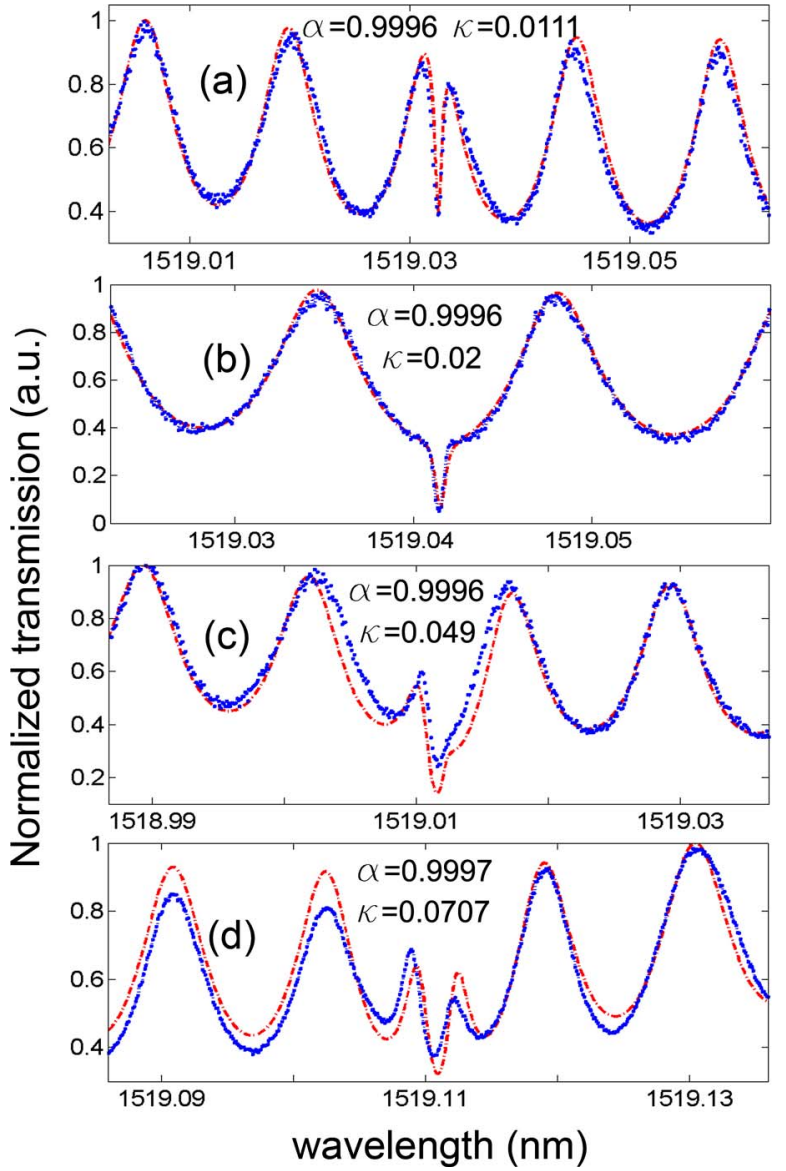

Fig. 3. (Color online) Measurements and theoretical fits of the transmission spectra of the microtoroid-FP coupledresonator system. Asterisk curves and dashed-dotted curves represent the measured and fitting data, respectively. Fitting parameters $\alpha$ and $\kappa$ are also given in (a)-(d).

resonator is then heat tapered to a diameter of $2 \mu \mathrm{m}$ and coupled to the microtoroid. We use a piezoelectric controller to modify the gap and thus coupling coefficient $\kappa$ between the microtoroid and the tapered fiber. Wavelength detuning is achieved by thermal tuning of the refractive index of the microtoroid. ${ }^{12}$ A tunable laser is used to launch the optical signal into the fiber, and a photodetector and an oscilloscope are used to measure the transmitted power as a function of the optical wavelength.

In Fig. 3 the experimentally measured normalized transmission spectra and the theoretical fits are plotted. Not all the line shapes in Fig. 2 can be measured because of difficulty in achieving the precise coupling strength and wavelength detuning. The parameters of the FP resonator used in the theoretical fitting are $L=3.0 \mathrm{~cm}, L_{\mathrm{FBG}}=3.4 \mathrm{~mm}$, and $\Delta n=10^{-4}$, and the propagation loss of the tapered fiber is assumed to be $\sim 0.38 \mathrm{~dB} / \mathrm{cm}$. Fitting parameters $\kappa$ and $\alpha$ are given in each case in Figs. 3(a)-3(d). Figures 3(a) and 3(b) represent the undercoupled regime for the on- and off-resonance cases, respectively. Figure 3(c) illustrates the intermediately coupled regime, and Fig. 3(d) represents the overcoupled regime. The line shapes of the transmission spectra agree well qualitatively and quantitatively with our theoretical prediction. Of particular interest, Fig. 3(c) exhibits the Fano-like resonance feature, and in Fig. 3(d) we observe two extra transmission peaks (compared with the single FP transmission spectrum). Thus we achieve a wide variety of resonance spectral features simply by varying the interresonator coupling and resonance detuning.

In summary, the coupled microtoroid-FP resonator system involves three-state coupling and manifests a variety of transmission line shapes. This implies that a variety of phase responses are possible, which could be important for controlling the phase or propagation velocity of an optical signal.

We thank Yong Xu and Reginald K. Lee for their generous help. This work is supported by the National Science Foundation (DMR-0120967) and the Defense Advanced Research Projects Agency (N00014-04-1-0094). J. K. S. Poon is grateful for financial support from the Natural Sciences and Engineering Research Council of Canada. Y. Huang's e-mail address is yanyi@caltech.edu.

\section{References}

1. K. J. Vahala, Nature 424, 849 (2003).

2. A. Yariv, IEEE Photon. Technol. Lett. 14, 483 (2002).

3. E. Krioukov, D. J. W. Klunder, A. Driessen, J. Greve, and C. Otto, Opt. Lett. 27, 512 (2002).

4. S. Fan, Appl. Phys. Lett. 80, 908 (2002).

5. C. Y Chao and L. J. Guo, Appl. Phys. Lett. 83, 1527 (2003)

6. D. K. Armani, T. J. Kippenberg, S. M. Spillane, and K. J. Vahala, Nature 421, 925 (2003).

7. Y. Xu, Y. Li, R. K. Lee, and A. Yariv, Phys. Rev. E 62 , 7389 (2000).

8. A. Yariv, Optical Electronics in Modern Communications (Oxford U. Press, 1997).

9. A. Yariv, Electron. Lett. 36, 321 (2000).

10. H. A. Haus, Waves and Fields in Optoelectronics (Prentice-Hall, 1984).

11. U. Fano, Phys. Rev. 124, 1866 (1961).

12. M. L. Gorodetsky and I. S. Grudinin, J. Opt. Soc. Am. B 21, 697 (2004). 